\title{
The Long and Winding Road of Vagus Nerve Stimulation: Challenges in Developing an Intervention for Difficult-to-Treat Mood Disorders
}

This article was published in the following Dove Press journal:

Neuropsychiatric Disease and Treatment

\author{
Harold A Sackeim (D) \\ Maxine Dibué ${ }^{2,3}$ \\ Mark T Bunker ${ }^{4}$ \\ A John Rush ${ }^{5-7}$
}

'Departments of Psychiatry and Radiology, Vagelos College of Physicians and Surgeons, Columbia University, New York, NY, USA; ${ }^{2}$ Department of Neurosurgery, Heinrich Heine University, Düsseldorf, Germany; ${ }^{3}$ Medical Affairs Europe, LivaNova Deutschland $\mathrm{GmbH}$, Munich, Germany; ${ }^{4}$ LivaNova USA PLC, Houston, TX, USA; ${ }^{5}$ Duke-NUS Medical School, Singapore; ${ }^{6}$ Department of Psychiatry and Behavioral Sciences, Duke University, Durham, NC, USA;

${ }^{7}$ Department of Psychiatry, Texas Tech University, Permian Basin, TX, USA
Correspondence: Harold A Sackeim Departments of Psychiatry and Radiology, Vagelos College of Physicians and

Surgeons, Columbia University, 2124

Moselem Springs Road, Fleetwood, PA

10522, USA

Tel + 917 273-5379

Email hasI@columbia.edu
Purpose: The Vagus Nerve Stimulation (VNS) Therapy System has been studied for more than 20 years in patients with severe, treatment-resistant, chronic mood disorder, i.e., difficult-to-treat depression (DTD). This review distills some of the implications of this research for future therapeutic trials in this population.

Methods: A narrative review is provided on VNS in DTD. Protocols for a new, large, shamcontrolled trial and a global, longitudinal observational study are described.

Results: Following encouraging results in open studies, a randomized, masked, shamcontrolled trial of VNS for DTD failed to demonstrate an effect on the primary outcome. The negative results may have been partly due to inadequate treatment duration (10 weeks). In long-term observational studies, adjunctive VNS, combined with treatment-as-usual (VNS +TAU), was administered to more than 1100 DTD patients and compared with TAU alone in more than 400 patients. VNS+TAU had superior antidepressant effects, but maximal symptom reduction was often observed after 12 months or longer of stimulation. VNS+TAU had also marked superiority in durability of benefit. Sustained levels of symptom reduction below the traditional cutoff for response (i.e. $<50 \%$ ) were associated with improved quality of life. Limitations: Most comparisons of VNS+TAU and TAU were derived from observational, open label studies.

Conclusion: The history of VNS in DTD has implications for interventional studies in this population, and perhaps other chronic medical disorders. The slow onset of benefit with VNS necessitates considerably longer controlled observation periods to establish efficacy. Durability of benefit should be routinely incorporated in efficacy assessment. New outcome metrics are needed to both categorically identify clinically meaningful benefit and to integrate information on symptom burden over time.

Keywords: vagus nerve stimulation, major depressive episode, difficult-to-treat depression, treatment-resistant depression, antidepressant, efficacy, regulatory process

\section{Introduction}

There has been explosive growth in the brain stimulation interventions available for psychiatric disorders and particularly unipolar and bipolar depression. ${ }^{1}$ The shortterm efficacy and safety of transcranial magnetic stimulation (TMS) is now well established, ${ }^{2-4}$ with impressive response and remission rates for major depressive episodes (MDE) in routine clinical use. ${ }^{5,6}$ In recent years, electroconvulsive therapy (ECT), the oldest biological treatment in continuous use in psychiatry, has undergone improvements that have dramatically reduced its acute and long-term cognitive effects, while preserving efficacy. ${ }^{7}$ 
ECT is now joined by non-invasive brain stimulation interventions, such as TMS, that are mainly considered for less severe, resistant, or chronic major depressive episodes, and by invasive forms of neuromodulation that are intended for more difficult-to-treat patients, who often have not benefited from or sustained benefit following multiple treatments, including ECT. ${ }^{8}$ The concept of difficult-to-treat depression (DTD) has been offered recently to highlight the fact that, amongst patients with treatmentresistant depression (TRD), a substantial subgroup do not initially attain or subsequently sustain response, let alone remission, regardless of the intensity and adequacy of antidepressant treatment. ${ }^{9,10}$ For these patients, long-term disease management strategies are of central importance, and treatments that offer sustained benefit are of particular interest. Testing the efficacy of new interventions in DTD is challenging. Few clinical trials examine clinical outcomes integrated over time, even in chronic medical disorders with a fluctuating course, e.g., diabetes mellitus, epilepsy, cardiovascular and neurodegenerative disorders. ${ }^{11,12}$ Furthermore, the traditional metrics for designating clinical benefit in standard trials of antidepressant medications may be problematic in DTD, such as classifying clinical response based on a $50 \%$ or greater reduction in depressive symptom severity. Recent evidence suggests that smaller degrees of symptomatic improvement in DTD result, nonetheless, in clinically meaningful improvements in quality of life. ${ }^{13}$

The Vagus Nerve Stimulation (VNS) Therapy System is currently the only invasive neurostimulation intervention approved by the US Food and Drug Administration for TRD. ${ }^{14}$ It fundamentally differs from ECT and TMS as it displays a delayed onset of therapeutic effect, as the percentage of patients meeting traditional response criteria incrementally increases over a one-year or longer stimulation period. ${ }^{15-21}$ Furthermore, those patients who show clinical benefit are likely to maintain it over long periods of time. ${ }^{18,21-24}$ These characteristics of VNS, slow onset and strong durability, pose challenges in designing clinical trials that adequately quantify its beneficial and adverse effects. Early studies of VNS in TRD used trial designs mainly based on acute treatment phase studies of antidepressant medications, applying the same pharmacological rationale to neuromodulation but without insight into the "electrodynamics" and "electrokinetics" of VNS or neuromodulation in general.

This report presents a narrative review of investigations of VNS in DTD with an emphasis on the implications of this history for designing clinical trials that evaluate the effectiveness and safety of new interventions in this population, where sustained remission may be rarely achieved and where more realistic goals often entail lesser degrees of sustained benefit. This review is restricted to investigations of left cervical VNS in MDE patients, in whom a pulse generator was surgically implanted in the chest wall that delivered electrical signals to a bipolar electrode attached to the left vagus nerve in the neck. In recent years, non-invasive transcutaneous forms of VNS (tVNS) have been developed, using surface electrodes to target the auricular branch of the vagus nerve in the ear or the cervical branch in the neck. ${ }^{25,26}$ However, clinical trials of tVNS in MDE patients have been largely non-randomized, small sample studies in populations without known treatment resistance. ${ }^{27-30} \mathrm{~A}$ clinical role for tVNS in MDE has yet to be established.

\section{Uncontrolled, Open-Label, Pilot Investigations}

Interest in VNS as a potential treatment for DTD was first prompted by observations regarding its use in epilepsy. In the early 1990s it was established that repetitive peripheral stimulation of the vagus nerve could arrest seizures in animal models of epilepsy, ${ }^{31,32}$ and subsequently that VNS was effective in improving seizure control in patients with medication-resistant epilepsy. ${ }^{33-35}$

These findings were of special interest, as it had long been argued that ECT's anticonvulsant properties, triggered by the seizure termination process, were integral to its efficacy in mood disorders, with the hypothesis that the anticonvulsant effects of ECT reflected enhanced GABAergic inhibition. ${ }^{36-38}$ The critical role of anticonvulsant medications in the acute and long-term management of mood disorders underscored the possibility that the long-term anticonvulsant effects of VNS might also be of benefit to some patients with DTD. ${ }^{39,40}$

Another consideration that prompted interest in VNS in DTD was the fact that stimulation of cranial nerves provides a unique method for modulating deep brain nuclei using peripheral stimulation. ${ }^{41}$ It is estimated that approximately $80 \%$ of the fibers stimulated with left cervical VNS are afferent to the brain. ${ }^{42}$ The first major intracerebral relay with cervical VNS is the nucleus tractus solitarius in the brain stem, whose widespread projections result in modulation of the locus ceruleus, dorsal raphe, hypothalamus, thalamus, amygdala, hippocampus, and multiple cortical regions. ${ }^{43,44}$ VNS had shown antidepressant properties in animal models 
of depression, ${ }^{45}$ and encouraging effects on mood in epilepsy patients. ${ }^{46}$ Thus, VNS offered a promising new method to impact on mood disorders by modulating deep brain nuclei with subsequent midbrain and cortical effects, in essence, a novel bottom-up approach to the treatment of mood disorders.

From the outset, it was recognized that VNS would be reserved for patients who had profound mood disturbance despite an extensive history of intensive antidepressant treatment, as it involved a surgical intervention, implanting a vagus nerve stimulator in a subcutaneous pocket in the chest area connected to a stimulating electrode which is wrapped around the vagus nerve in the neck. The first (pilot) investigation of VNS in TRD was the prospective, open-label D-01 study (see Table 1). ${ }^{16,17}$ (The "D-" prefix in the study identifier indicates that the study population comprised depressed patients, and the subsequent number identifies the particular study within this indication.) This study enrolled 60 patients across four US centers. Inclusion criteria required that the current MDE be at least 2 years in duration, or that patients had at least four lifetime MDEs. Treatment history was documented using the Antidepressant Treatment
History Form (ATHF), ${ }^{47,48}$ with the requirements that patients had not benefited from adequate treatment with at least two antidepressant medications from different classes, and from at least one course of psychotherapy. In fact, in these DTD patients the average duration of the current major depressive episode (MDE) was 10 years, the average number of documented antidepressant treatment trials in the current MDE was approximately 16 , and the average baseline score on the 28-item Hamilton Rating Scale for Depression $\left(\mathrm{HRSD}_{28}\right)$ was 37 , even though no patient met criteria for psychotic depression. In this first study, 40 of the $60(67 \%)$ patients had a history of treatment with ECT.

Prior to this pilot study, the industry sponsor (Cyberonics, Inc.) asked a group of mood disorder experts what they would accept as a minimal response rate at one year that would support further exploration of VNS in TRD. The consensus was that to continue clinical trials at least $30 \%$ of the sample should be categorized as responders at one year. Thus, from the outset it was anticipated that the number of patients who might benefit could be relatively modest, and that in this population, achievement of sustained clinical benefit, as opposed to complete

Table I Clinical Trials and Meta-Analyses of Vagus Nerve Stimulation (VNS) in the Treatment of Major Depressive Episodes

\begin{tabular}{|c|c|c|c|c|c|}
\hline Study & Publication & $\begin{array}{l}\text { VNS } \\
+ \text { TAU } \\
\text { (N) }\end{array}$ & $\begin{array}{l}\text { TAU } \\
(\mathbf{N})\end{array}$ & Design & $\begin{array}{l}\text { Study Duration } \\
\text { and Follow-Up }\end{array}$ \\
\hline D-0I & $\begin{array}{l}\text { Rush et al. }(2000) ;^{16} \\
\text { Sackeim et al. }(2001)^{17}\end{array}$ & 60 & - & Open-label, prospective, single arm & 24 months \\
\hline D-02 & Rush et al. $(2005)^{15}$ & 235 & - & $\begin{array}{l}\mathrm{RCT} \text {, sham-controlled ( } 10 \text { weeks), } \\
\text { then single arm, open label }\end{array}$ & 24 months \\
\hline D-03 & $\begin{array}{l}\text { Schlaepfer et al. }(2008)^{49} \\
\text { Bajbouj et al. }(2010)^{50}\end{array}$ & 74 & - & Open-label, prospective, single arm & 24 months \\
\hline D-04 & George et al. $(2005)^{51}$ & & 124 & Open-label, prospective, single arm & 24 months \\
\hline D-2I & Aaronson et al. $(2013)^{75}$ & 331 & - & RCT of VNS dosing (3 arms) & 12 months \\
\hline D-23 & Aaronson et al. $(2017)^{18}$ & 335 & 301 & $\begin{array}{l}\text { Open label, two arm, prospective } \\
\text { registry }\end{array}$ & 60 months \\
\hline Meta-analysis & Berry et al. $(2013)^{20}$ & 1035 & 425 & $\begin{array}{l}\text { Meta-analysis of D-0I, D-02, D-03, } \\
\text { D-04, D-2I, D-23 }\end{array}$ & - \\
\hline $\begin{array}{l}\text { Combined D-2I and D-23 } \\
\text { with } 5 \text { year follow-up }\end{array}$ & Aaronson et al. $(2017)^{18}$ & 494 & 301 & $\begin{array}{l}\text { Open label, two arm, prospective } \\
\text { registry }\end{array}$ & 60 months \\
\hline Meta-analysis & Bottomley et al. $(2020)^{19}$ & $|17|$ & 409 & Meta-analysis of 22 studies & - \\
\hline
\end{tabular}

Notes: The D-prefix in the study identifier indicates that the study population comprised depressed patients, and the subsequent number identifies the particular study within this indication. For example, D-0I was the first, pilot study of VNS in treatment-resistant depression using the NeuroCybernetic Prosthesis (NCP $\left.{ }^{\circledR}\right)$ System (LivaNova USA, Inc.).

Abbreviations: VNS, vagus nerve stimulation; TAU, treatment-as-usual; RCT, randomized controlled trial. 
remission, might meaningfully improve quality of life, and, perhaps, offer the best achievable result. All patients in this initial and all subsequent studies of VNS continued to receive treatment-as-usual (TAU), alone or combined with VNS. In other words, VNS was adjunctive to otherwise unconstrained treatment of the depression. Indeed, in this first pilot study 18 of 59 evaluable patients $(30.5 \%)$ met response criteria at one year. ${ }^{16,17}$

A similar multi-site, open label, prospective, observational study (D-03) in 74 MDE patients was later conducted in the European Union. ${ }^{49,50}$ Like the D-01 study participants, patients receiving VNS+TAU in D-03 showed progressive improvement over the first year. The response and remission rates after 3 months of VNS and TAU were $37 \%$ and $18 \%$, respectively. After one year, these rates were $53 \%$ and $33 \%$, respectively. In this study, the average time to achieve response status was 9 months.

\section{Randomized, Sham-Controlled and Treatment-as-Usual Studies Leading to FDA Approval}

The D-01 and D-03 pilot studies were uncontrolled and open label. Patients received TAU in addition to VNS, raising the possibility that alterations in treatment regimens, other than the addition of VNS, were to some extent responsible for the observed clinical improvement. The industry sponsor used the findings of D-01 to design a multi-site, randomized, blinded, sham-controlled, pivotal D-02 trial that it hoped would support FDA regulatory approval of VNS for DTD (ClinicalTrials.gov Identifier: NCT00533832). This study examined antidepressant effects for up to 2 years following implantation. However, the blinded, randomized sham-controlled phase was only 10 weeks in duration (during the first two of which the VNS settings were gradually titrated upward), followed by single arm, open label, active treatment of all 235 patients. $^{15,51,52}$

The primary outcome measure in this pivotal trial was the rate of response based on $\mathrm{HRSD}_{28}$ scores at the end of the 10-week, randomized, sham-controlled phase. This aspect of the design paralleled the methods used in shortterm, placebo-controlled, randomized clinical trials commonly used to establish the acute treatment phase efficacy with antidepressant medications. ${ }^{53}$ At the end of the shamcontrolled period, adjunctive VNS with treatment as usual (VNS+TAU) had a $15 \%$ response rate compared with $10 \%$ in the sham control group, a non-significant difference. Thus, the study had failed to provide compelling Class I evidence regarding the efficacy of VNS over the 10-week period. The short duration of controlled treatment prior to the primary outcome time point likely contributed to the failure to observe a treatment effect. In addition, mean VNS current $(0.67 \mathrm{~mA})$ during the masked 10 -week treatment period was considerably lower than the recommended current setting of $1-2 \mathrm{~m} .^{54}$

Positive findings from this trial provided grounds for arguing, however, that VNS had an important therapeutic role in the management of DTD. First, there was a significant difference between the blinded sham and active VNS conditions at the 10-week time point in selfreported depressive symptom severity (IDS-SR), ${ }^{55}$ a secondary outcome measure. Second, after 12 months of VNS, antidepressant effects further improved, much like the progressive improvement observed in the D-01 and later D-03 studies. ${ }^{52}$ At one year, approximately $30 \%$ of patients were classified as responders, whether based on $\mathrm{HRSD}_{28}$, Montgomery Åsberg Depression Rating Scale (MADRS), ${ }^{56}$ or Clinical Global Impression Improvement $(\mathrm{CGI}-\mathrm{I})^{57}$ ratings. Third, to provide long-term comparator data, a parallel TAU sample was collected and termed the D-04 study (see Table 1). Patients enrolled in the D-04 study were treated generally at the same sites and had similar clinical and treatment history characteristics as the D-02 sample, but they did not receive adjunctive VNS. ${ }^{51,58}$

The comparison with the non-randomized 12-month TAU group from the D-04 study showed that VNS+TAU resulted in superior antidepressant effects than TAU alone both over the entire 12-month period and in response rates at one year. $^{51}$ In essence, it appeared that the clinical benefits of VNS accrued over a longer time period than had ever been seen with a pharmacological or another neuromodulation intervention for MDE. The shamcontrolled period was simply too short to adequately test the efficacy of VNS.

With the completion of the D-02 and D-04 studies, the industry sponsor prepared a submission to the FDA seeking clearance of adjunctive VNS in DTD, despite the negative findings from the sham-controlled phase. While this material was being prepared, the data on durability of benefit of VNS were examined. ${ }^{22,23}$ Clearly, antidepressant interventions are of limited value if their benefits are only transient, as is the case with sleep deprivation. ${ }^{59,60}$ Establishing sufficient durability of benefit is especially 
at issue in DTD. The findings from the Sequenced Treatment Alternatives to Relieve Depression (STAR*D) study later demonstrated that with increasing levels of prospectively identified treatment resistance there is both a reduced probability of remitting with a new treatment and, if remission is achieved, an increased probability of relapse and loss of benefit during the subsequent year. ${ }^{61}$ Indeed, the conjoint probability of both remitting and sustaining the remission for one year was less than $5 \%$ at the third and fourth levels of STAR*D. ${ }^{62}$ As noted, there is similar evidence in ECT samples where the degree of medication resistance has been found to predict both acute ECT clinical outcome ${ }^{63-65}$ and post-ECT relapse rate. ${ }^{66-69}$

The findings on durability of benefit were striking, and provided strong evidence that VNS was a clinically valuable intervention in DTD. Across the D-01 and D-02 studies, early responders (within 3 months of starting VNS) and late responders (responders at 12 months and not 3 months) were identified. ${ }^{22}$ For the early responders, outcomes at 12 months and 24 months were examined, while 24-month outcomes were examined for late responders. For early responders, $63-72 \%$ showed substantial clinical benefit $(\geq 40 \%$ improvement in HRSD scores relative to baseline) at 12 months, and these rates were $72-77 \%$ at 24 months. Similarly, $65-79 \%$ of late responders continued to show substantial clinical benefit at 24 months. These high rates of continued improvement were maintained throughout the follow-up periods. Analyses of serial symptom scores, both clinician-rated and self-report, also demonstrated that symptomatic improvement, once achieved, was generally stable throughout follow-up. In other words, despite their extraordinary levels of illness severity, chronicity, and treatment resistance, patients in the D-01 and D-02 studies who benefited from VNS sustained their clinical gains at remarkably high rates. The fact that VNS is administered continuously, and without issues of compliance, may have contributed to its notable durability of benefit. ${ }^{22}$ It has also been hypothesized that mood disorder treatments with relatively slow onset of therapeutic action, like VNS, may exhibit greater persistence of benefit than interventions where clinical benefit is rapidly achieved, e.g., ketamine or ECT. ${ }^{70}$

The FDA convened an advisory panel in June, 2004 to review the evidence regarding VNS in DTD and included individuals with expertise in psychopharmacology and ECT. After presentation of the evidence, the vote was split, with the majority of the panel recommending FDA approval of VNS for this indication. However, despite the positive recommendation of the advisory panel, the FDA issued a preliminary non-approval letter. The absence of Class I evidence unequivocally demonstrating superior efficacy relative to sham treatment was considered an insurmountable limitation. Ultimately, the long-term data demonstrating the sustained clinical benefit as compared with TAU and the findings of strong durability of benefit led to a re-assessment of the application. On July 15, 2005, the FDA issued a final ruling, stating that

The VNS Therapy System is indicated for the adjunctive longterm treatment of chronic or recurrent depression for patients 18 years of age or older who are experiencing a major depressive episode and have not had an adequate response to four or more adequate antidepressant treatments. ${ }^{71}$

It appeared that the absence of alternative treatments known to be effective for DTD, and the evidence regarding durability of benefit were particularly influential.

As has become apparent, regulatory approval or clearance of a medical device does not guarantee that public or private insurers will reimburse patients or providers. On May 4, 2007, the Centers for Medicare and Medicaid Services (CMS) issued a non-coverage determination for VNS Therapy in TRD on the basis of insufficient evidence of efficacy. ${ }^{72}$ At the heart of the CMS' reservation was the failure of the sham-controlled D-02 study to unequivocally demonstrate efficacy. The rejection of coverage by CMS was followed by non-coverage policies issued by private insurers. Since 2005, in the United States VNS has been a FDA-approved treatment for DTD, but has lacked coverage by government and commercial payers. Since the costs of the device and its surgical implantation are significant, VNS has been out of the reach of DTD patients unless they could self-pay or received the treatment pro bono as part of a research protocol. This uncertainty about the merits of VNS in DTD, as expressed by CMS, also has been reflected in inconsistent policies among European and other countries regarding the use of VNS in DTD, often leading to barriers in access. ${ }^{73,74}$

Most clinicians who are aware of the history of VNS therapy development may recall a period of initial excitement about a potential new treatment for DTD, the convoluted path to FDA approval and then the subsequent disappointment with the denial of insurance coverage. Fortunately, since then there have been two major developments that have generated renewed interest in VNS for this population. 


\section{Post-Approval Randomized Trial and Registry Studies}

First, research on VNS in TRD continued after the D-02 pivotal trial. The D-21 study constituted a second multisite, double-blind, randomized trial of VNS (ClinicalTrials.gov Identifier: NCT00305565). ${ }^{75}$ In this study, 310 DTD patients were randomly assigned to three different doses of VNS: low dose (output current of 0.25 $\mathrm{mA}$; pulse width of $130 \mu \mathrm{s}$ ), medium dose (output current of $0.5-1.0 \mathrm{~mA}$, pulse width of $250 \mu \mathrm{s}$ ), and high dose (output current of 1.25-1.5 mA, pulse width of $250 \mu \mathrm{s}$ ). The FDA had requested this post-marketing study, since a dose finding investigation had not been conducted in DTD, and stimulation parameters in previous DTD research were largely informed by the research in epilepsy.

The D-21 study provided another opportunity to establish the efficacy of VNS in DTD under randomized, controlled conditions. It was hypothesized that the low dose condition would be less effective than either the moderate or high dose condition, with the reduced therapeutic properties due to a weak level of stimulation. Indeed, in the field of ECT, it is well established that manipulations of electrical dosing and electrode placement strongly influence the efficacy of the intervention. ${ }^{67,76-78}$ In many respects, the demonstrations that such technical factors strongly determine ECT clinical outcome provide stronger evidence of ECT efficacy than sham-controlled (anesthesia alone) trials. Similarly, the D-21 trial, in addition to investigating optimal VNS dosing, was intended to provide key data regarding the efficacy of the intervention. The fact that dose-response relations had been demonstrated for VNS in epilepsy provided further grounds for the D-21 study. ${ }^{79}$

The randomized controlled phase in D-21 lasted for 22 weeks, a longer time frame than D-02, and after this phase, dosing could be optimized for each patient with outcome assessment continuing until 50 weeks. Across all three dosage groups, significant antidepressant effects were observed at the end of the 22-week acute phase, but with additional symptom reduction occurring at the end of the 50-week long-term phase. Contrary to the hypothesis, the three dosage groups did not differ in antidepressant efficacy at the end of the 22-week acute phase. Rather, across patients (ignoring the treatment assignments), the total electrical intensity (charge) administered per day was related to symptom severity: a higher daily charge was associated with greater depressive symptom reduction.
Furthermore, patients in the medium and high dosage groups who were responders at the end of the acute phase (22 weeks) were significantly more likely to maintain response at the 50 -week time point than patients randomized to the low dosage group.

In retrospect, the failure to demonstrate a doseresponse separation at the end of the acute phase might be attributable to two factors. As in the preceding research on VNS in DTD, clinical improvement continued through the 50-week study period, so the 22-week acute phase did not fully capture this phenomenon. Second, the randomization of patients to different device output intensities did not account for individual differences in dosing needs. It is routine with neuromodulation interventions such as TMS and ECT to titrate the stimulation intensity for each individual relative to a neurophysiological biomarker criterion. For example, in TMS, the intensity of repetitive magnetic pulses is usually based on the intensity of a single magnetic pulse required to reliably evoke a response from the representation of a specific muscle in motor cortex (e.g., motor evoked potential or finger twitch). ${ }^{80,81}$ Similarly in ECT dosing is relative to the seizure threshold, often identified for each individual using an empirical titration procedure, where electrical intensity is progressively increased until a generalized seizure is evoked. ${ }^{82,83}$ In VNS, individual differences in vagus nerve anatomy, positioning of the stimulating electrode, effective stimulation area, tissue conductivity, and other factors may have substantial impact on the afferent stimulation. The variability introduced by these individual difference factors may obscure relations with absolute stimulus intensity, as defined by the treatment group assignments in the D-21 study. For example, in ECT, absolute electrical dosage (charge) does not correlate with efficacy or cognitive side effects, while electrical dosage relative to the individual's seizure threshold shows consistent associations. ${ }^{76,83}$ In the absence of a biomarker to guide VNS dosing, the existing practice is to progressively increase the current of the VNS pulse to the maximally tolerated intensity, essentially titrating against side effects. Indeed, the secondary findings in D-21 suggested that higher dosage levels produce stronger and more durable therapeutic effects.

As another condition of the original FDA approval, a registry was implemented in which DTD patients treated with VNS+TAU were followed for up to 5 years, as well as comparable patients who received only TAU. In the D-23 study, 335 patients who received VNS+TAU and 
301 patients who received only TAU were enrolled in the registry (ClinicalTrials.gov Identifier: NCT00320372). Additionally, 159 patients from D-21 who received VNS +TAU were also followed as registry participants. Aaronson et al. ${ }^{18}$ examined the clinical outcomes over a 5-year period of 494 patients treated with adjunctive VNS and 301 TAU-treated patients (see Table 1).

The 5-year cumulative first-time response $(67.6 \%$ vs $40.9 \%$ ) and remission (43.3\% v. $25.7 \%$ ) rates were greater in the VNS+TAU group compared with TAU alone. The adjunctive VNS group also had advantages in time to response and remission (shorter with VNS) and time to recurrence (longer with VNS). This therapeutic superiority of adjunctive VNS compared with TAU was consistent across unipolar and bipolar depression subtypes, and patients with and without a co-morbid generalized anxiety disorder, and the magnitude of the effects appeared clinically meaningful. Of note, the advantage of VNS+TAU over TAU alone in long-term outcome pertained both to patients with a history of positive ECT response and those who did not appear to benefit from ECT.

A separate report based on the D-23 registry data examined durability of benefit. ${ }^{24}$ Patients receiving VNS +TAU who met response criteria were less likely to relapse than TAU only responders. The interval between response and relapse was also longer among VNS+TAU responders than TAU only responders. Fast or slow onset of response was not predictive of its durability.

\section{Meta-Analyses of the VNS Literature in DTD}

Several comprehensive reviews and meta-analyses have been reported on VNS in DTD. ${ }^{19,20,84-86}$ Berry et al. ${ }^{20}$ conducted a meta-analysis across the studies listed in Table 1, involving 1035 patients treated with VNS+TAU and 425 patients treated only with TAU. Bottomley and colleagues ${ }^{19}$ more broadly examined 22 studies of VNS or TAU in their meta-analysis, including retrospective case series. They compared outcomes in 1171 patients treated with VNS+TAU and 409 patients treated with only TAU.

The findings of these two meta-analyses were consistent. Patients treated with VNS+TAU were approximately three times more likely to achieve response and nearly five times more likely to achieve remission than patients treated with TAU only. The onset of clinical benefit was often slow, with twice as many patients classified as responders after one year of VNS compared with 6 months of treatment, and there was evidence of continued improvement up to 2 years after implantation. The one-year response rate with VNS+TAU ranged from 30-40\%, compared with a $12-18 \%$ response rate with TAU. In addition, one of the meta-analyses also provided evidence on the issue of durability. ${ }^{20}$ In patients who were responders at 24 weeks the likelihood of sustained response at 96 weeks was nearly 3.5 times greater in those treated with VNS +TAU compared with TAU alone.

\section{The RECOVER Study}

The D-23 VNS registry contains the largest prospective sample ever collected of patients with documented TRD and the longest follow-up of patients treated for episodes of major depression. The findings that have emerged in recent years have affirmed the therapeutic superiority of VNS+TAU compared with TAU alone. In particular, it appears that relative to TAU alone, patients receiving $\mathrm{VNS}+\mathrm{TAU}$ are considerably more likely to both achieve meaningful clinical benefit and to sustain that benefit. However, these conclusions derive mainly from open label, non-randomized comparisons and case series. Given the costs of the procedure, the promise of VNS in DTD may remain unfulfilled unless the coverage issue is addressed.

The second development prompting renewed interest in VNS for DTD may resolve the problem with coverage. The evidence emerging from the D-23 study prompted renewed interest from CMS in evaluating VNS for DTD. First, CMS convened an expert panel [Medicare Evidence Development and Coverage Advisory Committee (MEDCAC)] in April 2016 to determine whether patients with DTD/TRD can be accurately identified and their outcomes reliably assessed. Both prerequisites were confirmed by the panel and also later by a CMScommissioned technology assessment, sponsored by the Agency for Healthcare Research and Quality. ${ }^{87}$ The industry sponsor filed a formal request for reconsideration to CMS in October 2017 and, in February 2019, CMS issued a National Coverage Determination regarding VNS. ${ }^{88}$ CMS stipulated that it would

cover FDA approved vagus nerve stimulation (VNS) devices for treatment resistant depression (TRD) through Coverage with Evidence Development (CED) when offered in a CMS approved, double-blind, randomized, placebo-controlled trial with a follow-up duration of at least one year. 
Through the CED mechanism, CMS committed significant financial resources to support a definitive trial that could provide the critical Class 1 evidence needed to recommend or deny coverage by Medicare for VNS for DTD. On September 4, 2019, CMS approved such a trial with LivaNova as the sponsor (ClincalTrials.gov Number: NCT03887715).

The RECOVER (A PRospective, Multi-cEnter, Randomized Controlled Blinded Trial DemOnstrating the Safety and Effectiveness of VNS Therapy ${ }^{\circledR}$ System as AdjunctivE Therapy Versus a No Stimulation Control in Subjects With Treatment-Resistant Depression, clinicatrials.gov NCT03887715) trial protocol has been published, ${ }^{89}$ and the first patient enrolled on September 26, 2019. Up to 1100 DTD patients (to yield up to 500 patients with unipolar and 500 patients with bipolar disorder - all in an MDE upon entry) will be recruited at up to 100 US sites. The trial has three key and unusual features.

First, unlike many Medicare beneficiaries, patients with DTD are typically younger than 65 years old and qualify for Medicare benefits after being found eligible for Social Security Disability. In essence, CMS coverage defines a DTD sample that has marked and longstanding functional impairment due to their mood disorder, in addition to well documented treatment-resistance and illness chronicity.

Second, the trial design randomizes patients to active VNS+TAU or sham VNS+TAU (implantation but no stimulation) for one year. After this blinded, randomized, sham-controlled phase, all patients receive active VNS and participate in a long-term registry that documents outcomes for 5 years following implantation (4 years following the RCT). A one-year period of sham treatment is without precedent. This duration was selected given the slow and progressive rate of improvement consistently seen with VNS in DTD ${ }^{17,18,52}$ and the desire to address lessons from past studies whose randomized controlled phase was too brief. Of note, patients in both the sham and active groups receive TAU. This means that, while significant treatment alterations are discouraged, patient providers can nonetheless offer alternative treatments they deem advisable during the study, including maintenance ECT. The one-year, controlled period is a compromise. A longer period after surgical implantation in which active treatment with VNS is withheld would be dubious on ethical grounds. However, the longest feasible randomized controlled period is likely the most informative, given that the differences between VNS +TAU and TAU progress over more than a year and are then maintained.

Third, the outcomes metrics used in this trial are distinct from those used in short-term trials of antidepressant medications and neuromodulation interventions. Most commonly, antidepressant efficacy studies contrast treatment groups in depression symptom severity scores or rates of response or remission at a single acute study endpoint. With VNS in DTD it is evident that clinical benefit accrues over a long and quite variable period of time. Furthermore, DTD patients may benefit substantially from degrees of symptomatic improvement that fall short of remission, and remission may be elusive or fleeting for the majority of these patients. It also appears that, at least with pharmacological treatment and ECT, degree of treatment resistance predicts likelihood of relapse once benefit is obtained. Consequently, in DTD it would seem imperative on both scientific and clinical grounds to integrate information on the likelihood of clinically meaningful improvement with the durability of this improvement when deriving outcome measures.

The primary outcome in the RECOVER study is the percent of time during the 12-month randomized controlled phase during which the patients met criteria for response on the MADRS. ${ }^{56}$ In essence this metric combines information on the achievement of the outcome and its duration during the key study period. Secondary outcomes include the time to first attaining response (and remission) and the maximum consecutive period in response (and remission). Each of these outcome measures combine attainment of a clinical goal with either a measure of its duration or the speed of attainment.

In parallel with the RECOVER study, additional real world data are needed to examine the effects of VNS on long-term outcomes in multiple domains impacted by DTD (e.g., work productivity, cognition, anxiety). A new longitudinal observational study, termed the RESTORELIFE trial (A Global PRospective, Multi-cEnter, ObServational Post-markeT Study tO Assess shoRt, Mid and Long-term Effectiveness and Efficiency of VNS Therapy as Adjunctive Therapy in reaL-world patIents With diFficult to Treat dEpression) (ClincalTrials.gov number: NCT03320304), aims to recruit, at approximately 80 centers globally, a minimum of 500 patients with DTD treated with VNS and document these outcomes over a period of up to 60 months. 


\section{Implications and Conclusions}

The findings over the past two decades of clinical trials of VNS informed the design of the RECOVER trial, and they have implications more broadly for the study of therapeutics in DTD. First, it is clear that antidepressant treatments radically differ in their speed of therapeutic action. On the one hand, marked therapeutic effects can be observed within hours of treatment with ketamine $^{90}$ or esketamine, ${ }^{91}$ within days with ECT, ${ }^{92,93}$ weeks with standard antidepressant medications ${ }^{94}$ or TMS, ${ }^{6}$ and months to years with VNS. ${ }^{19,20}$ Depending on the intervention, different controlled observation periods are needed to capture these differences in therapeutic action and to document maximal therapeutic effects.

Sham-controlled periods of 3 months have been used to evaluate the safety and efficacy of deep brain stimulation (DBS) to the internal globus pallidus nucleus for dystonia, ${ }^{95}$ the subthalamic nucleus in Parkinson's disease, ${ }^{96}$ the anterior nucleus of the thalamus for partial epilepsy, ${ }^{97,98}$ and various targets in obsessive-compulsive disorder. ${ }^{99}$ To our knowledge, a sham-controlled period of 12 months has never been attempted with a brain stimulation treatment. While the long latency for maximal therapeutic effect with VNS necessitates the long sham period, it undoubtedly introduces its own challenges. Maintenance of the blind for some patients may be problematic over such a long period, although there is no evidence that the blind would be more compromised over a 12-month period than a 3-month period. Indeed, dissociation between a progressive improvement in symptoms scores with no change in unblinding could provide evidence that symptomatic change was not attributable to patient knowledge of the treatment conditions. However, precisely because use of a long controlled observation period may challenge integrity of the patient blind, the primary outcomes measures in the RECOVER trial are completed by a team of off-site central raters who have no prior information about the study design, or the individual patient and their timepoint in the study.

Another implication of the prior investigations of VNS is that the measures of therapeutic success traditionally used in antidepressant treatment trials may be inappropriate when applied to DTD. ${ }^{9,10}$ In recent years, the goal of antidepressant treatment has generally shifted from the attainment of symptomatic response to symptomatic remission, as the extent of residual post-treatment symptoms is believed to negatively impact on durability of benefit and quality of life. ${ }^{100,101}$ However, in DTD remission may be rarely achieved, and only fleeting in persistence. Rather, in the VNS trials to date patients who achieved either response or remission showed strong durability of benefit. ${ }^{18,22,24}$ In these patients there was also evidence that degrees of symptomatic improvement less than the traditional threshold of $50 \%$ were associated with meaningful improvement in quality of life. ${ }^{13}$ It is noteworthy that the $50 \%$ criterion for declaring response is traditionally applied across all types of antidepressant treatment and depressed patient populations. It was derived largely from early experience with antidepressant medications and ECT in non-resistant, treatment-naïve samples. In schizophrenia and obsessive compulsive disorder, lower thresholds for symptom change are commonly used when designating response. New empirical investigations are needed to determine within DTD samples the minimum depressive symptom change associated with acceptable durability of benefit and persistent improvement in quality of life. ${ }^{21}$

Regulatory approval of antidepressant medications and medical devices to treat MDE are based mainly on the findings of short-term acute treatment trials. In patients with chronic and/or highly recurrent illness, short-term outcomes at a uniform time point may not be reliable efficacy indicators due to an admixture of variable time to achieve initial benefit, limited extent of benefit, and variation in the durability of benefit. ${ }^{102}$ Antidepressant interventions should be evaluated both in terms of the probability of achieving a benefit and the probability of sustaining that benefit once achieved, i.e., the conjoint probability of both obtaining and sustaining benefit. ${ }^{70}$ While VNS for DTD may benefit only a minority of patients when assessed by rate of initial response, its apparent strong durability of benefit is certainly a fundamental consideration in evaluating its overall efficacy. The conundrum, of course, is that durability of benefit is optimally assessed over a long time frame, perhaps a period of several years, which is impractical when using a sham- or placebo-controlled trial design.

Finally, the metrics used to quantify clinical outcomes may need rethinking. Across many chronic medical disorders, the most common outcome measures are "snapshots" of illness manifestations at specific time points. This is especially the case when categorical outcomes, like response and remission, are examined. Such snapshots do not necessarily provide reliable indicators of benefit, as would be obtained with repeated measurement. Furthermore, such snapshots fail to integrate information on symptom manifestation over time, and thus cannot 
adequately address the degree of sustained benefit associated with antidepressant interventions. New approaches need to be evaluated that integrate symptom manifestations over time, thereby providing new measures of the long-term and sustained impact of antidepressant treatments for DTD.

\section{Abbreviations}

CMS, Centers for Medicare and Medicaid Services; DBS, deep brain stimulation; DTD, difficult-to-treat depression; ECT, electroconvulsive therapy; FDA, Food and Drug Administration; MDE, major depressive episode; TMS, transcranial magnetic stimulation; TRD, treatmentresistant depression; VNS, vagus nerve stimulation.

\section{Acknowledgments}

Scott T. Aaronson, MD, Charles R. Conway, MD, R. Hamish McAllister-Williams, MD, PhD, Bryan Olin, $\mathrm{PhD}$, and Jonathan Walker, BSc provided valuable comments on earlier versions of the manuscript.

\section{Funding}

Preparation of this review was supported in part by LivaNova PLC.

\section{Disclosure}

Professor Sackeim serves as a scientific adviser to the MECTA Corporation and Neuronetics Inc., as well as LivaNova PLC, which manufactures the Vagus Nerve Stimulation Therapy ${ }^{\circledR}$ System. He receives honoraria and royalties from Elsevier, Inc. and Oxford University Press. He is the inventor on nonremunerative US patents for Focal Electrically-Administered Seizure Therapy (FEAST) (8712532), titration in the current domain in ECT (9789310), and the adjustment of current in ECT devices (10583188), each held by the MECTA Corporation. He is also the originator of magnetic seizure therapy (MST). Dr. Dibué is an employee of LivaNova PLC, manufacturer of vagus nerve stimulators and holds stock options. Dr Bunker is a former employee and a current consultant of LivaNova USA PLC. Professor Rush has received consulting fees from Akili, Brain Resource Inc., Compass Inc., Curbstone Consultant LLC, Emmes Corp., Holmusk, Johnson and Johnson (Janssen), LivaNova USA PLC, Neurocrine Biosciences Inc., Otsuka-US, and Sunovion; speaking fees from LivaNova; and royalties from Guilford Press and the University of Texas Southwestern Medical Center, Dallas, TX (for the Inventory of Depressive Symptoms and its derivatives). He is also named coinventor on two patents: US Patent
No 7,795,033: Methods to Predict the Outcome of Treatment with Antidepressant Medication, Inventors: McMahon FJ, Laje G, Manji H, Rush AJ, Paddock S, Wilson AS; and US Patent No 7,906,283: Methods to Identify Patients at Risk of Developing Adverse Events During Treatment with Antidepressant Medication, Inventors: McMahon FJ, Laje G, Manji H, Rush AJ, Paddock S. The authors report no other conflicts of interest in this work.

\section{References}

1. George MS, Sackeim HA. Brain stimulation, revolutions, and the shifting time domain of depression. Biol Psychiatry. 2008;64 (6):447-448. doi:10.1016/j.biopsych.2008.07.013

2. Perera T, George MS, Grammer G, Janicak PG, Pascual-Leone A, Wirecki TS. The clinical TMS society consensus review and treatment recommendations for TMS therapy for major depressive disorder. Brain Stimul. 2016;9(3):336-346. doi:10.1016/j. brs.2016.03.010

3. Mutz J, Vipulananthan V, Carter B, Hurlemann R, Fu CHY, Young AH. Comparative efficacy and acceptability of non-surgical brain stimulation for the acute treatment of major depressive episodes in adults: systematic review and network meta-analysis. BMJ. 2019;364:11079. doi:10.1136/bmj.11079

4. McClintock SM, Reti IM, Carpenter LL, et al. Consensus recommendations for the clinical application of repetitive transcranial magnetic stimulation (rTMS) in the treatment of depression. J Clin Psychiatry. 2018;79(1):35-48. doi:10.4088/JCP.16cs10905

5. Carpenter LL, Janicak PG, Aaronson ST, et al. Transcranial magnetic stimulation (TMS) for major depression: a multisite, naturalistic, observational study of acute treatment outcomes in clinical practice. Depress Anxiety. 2012;29(7):587-596. doi:10.1002/da.21969

6. Sackeim HA, Aaronson ST, Carpenter LL, et al. Clinical outcomes in a large registry of patients with major depressive disorder treated with transcranial magnetic stimulation. $J$ Affect Disord. 2020;277:65-74. doi:10.1016/j.jad.2020.08.005

7. Sackeim HA. Modern electroconvulsive therapy: vastly improved yet greatly underused. JAMA Psychiatry. 2017;74(8):779-780. doi:10.1001/jamapsychiatry.2017.1670

8. Gaynes BN, Lux L, Gartlehner G, et al. Defining treatment-resistant depression. Depress Anxiety. 2020;37(2):134-145. doi:10.1002/ da.22968

9. Rush AJ, Aaronson ST, Demyttenaere K. Difficult-to-treat depression: a clinical and research roadmap for when remission is elusive. Aust N Z J Psychiatry. 2019;53(2):109-118. doi:10.1177/00048 67418808585

10. McAllister-Williams RH, Arango C, Blier P, et al. The identification, assessment and management of difficult-to-treat depression: an international consensus statement. J Affect Disord. 2020; 267:264-282. doi:10.1016/j.jad.2020.02.023

11. National Academies of Sciences, Engineering, and Medicine: Health and Medicine Division: Food and Nutrition Board: Committee on the Development of Guiding Principles for the Inclusion of Chronic Disease Endpoints in Future Dietary Reference Intakes. Measuring chronic disease outcomesIn: Oria M, Kumanyika S, editors. Guiding Principles for Developing Dietary Reference Intakes Based on Chronic Disease. National Academies Press (US); 2017.

12. Working Group on Health Outcomes for Older Persons with Multiple Chronic Conditions. Universal health outcome measures for older persons with multiple chronic conditions. $J$ Am Geriatr Soc. 2012;60(12):2333-2341. doi:10.1111/j.1532-5415.2012.042 40.x. 
13. Conway CR, Kumar A, Xiong W, Bunker M, Aaronson ST, Rush AJ. Chronic vagus nerve stimulation significantly improves quality of life in treatment-resistant major depression. J Clin Psychiatry. 2018;79(5). doi:10.4088/JCP.18m12178

14. Food and Drug Administration. Summary of safety and effectiveness data (SSED) for vagus nerve stimulation in depression; July 13, 2020 [updated June 15, 2005]. Available from: https://www. accessdata.fda.gov/cdrh_docs/pdf/p970003s207b.pdf. Accessed November 30, 2020.

15. Rush AJ, Marangell LB, Sackeim HA, et al. Vagus nerve stimulation for treatment-resistant depression: a randomized, controlled acute phase trial. Biol Psychiatry. 2005;58(5):347-354. doi:10.1016/j.biopsych.2005.05.025

16. Rush AJ, George MS, Sackeim HA, et al. Vagus nerve stimulation (VNS) for treatment-resistant depressions: a multicenter study. Biol Psychiatry. 2000;47(4):276-286. doi:10.1016/s0006-3223 (99)00304-2

17. Sackeim HA, Rush AJ, George MS, et al. Vagus nerve stimulation (VNS) for treatment-resistant depression: efficacy, side effects, and predictors of outcome. Neuropsychopharmacology. 2001;25 (5):713-728. doi:10.1016/S0893-133X(01)00271-8

18. Aaronson ST, Sears P, Ruvuna F, et al. A 5-year observational study of patients With treatment-resistant depression treated with vagus nerve stimulation or treatment as usual: comparison of response, remission, and suicidality. Am J Psychiatry. 2017;174 (7):640-648. doi:10.1176/appi.ajp.2017.16010034

19. Bottomley JM, LeReun C, Diamantopoulos A, Mitchell S, Gaynes BN. Vagus nerve stimulation (VNS) therapy in patients with treatment resistant depression: a systematic review and meta-analysis. Compr Psychiatry. 2020;98:152-156. doi:10.10 16/j.comppsych.2019.152156

20. Berry SM, Broglio K, Bunker M, Jayewardene A, Olin B, Rush AJ. A patient-level meta-analysis of studies evaluating vagus nerve stimulation therapy for treatment-resistant depression. Med Devices (Auckl). 2013;6:17-35. doi:10.2147/ MDER.S41017

21. Trottier-Duclos F, Desbeaumes Jodoin V, Fournier-Gosselin MP, et al. A 6-year follow-up study of vagus nerve stimulation effect on quality of life in treatment-resistant depression: a pilot study. $J$ ECT. 2018;34(4):e58-e60. doi:10.1097/YCT.0000000000000 485

22. Sackeim HA, Brannan SK, Rush AJ, George MS, Marangell LB, Allen J. Durability of antidepressant response to vagus nerve stimulation (VNS). Int J Neuropsychopharmacol. 2007;10 (6):817-826. doi:10.1017/S1461145706007425

23. Nahas Z, Marangell LB, Husain MM, et al. Two-year outcome of vagus nerve stimulation (VNS) for treatment of major depressive episodes. J Clin Psychiatry. 2005;66(9):1097-1104. doi:10.4088/ jcp.v66n0902

24. Kumar A, Bunker MT, Aaronson ST, et al. Durability of symptomatic responses obtained with adjunctive vagus nerve stimulation in treatment-resistant depression. Neuropsychiatr Dis Treat. 2019;15:457-468. doi:10.2147/NDT.S196665

25. Yap JYY, Keatch C, Lambert E, Woods W, Stoddart PR, Kameneva T. Critical review of transcutaneous vagus nerve stimulation: challenges for translation to clinical practice. Review. Front Neurosci. 2020;14(284). doi:10.3389/ fnins.2020.00284

26. Kaniusas E, Kampusch S, Tittgemeyer M, et al. Current directions in the auricular vagus nerve stimulation $\mathrm{i}-\mathrm{a}$ physiological perspective. Review. Front Neurosci. 2019;13(854). doi:10.3389/ fnins.2019.00854

27. Hein E, Nowak M, Kiess O, et al. Auricular transcutaneous electrical nerve stimulation in depressed patients: a randomized controlled pilot study. J Neural Transm. 2013;120(5):821-827. doi:10.1007/s00702-012-0908-6
28. Fang J, Rong $\mathrm{P}$, Hong $\mathrm{Y}$, et al. Transcutaneous vagus nerve stimulation modulates default mode network in major depressive disorder. Biol Psychiatry. 2016;79(4):266-273. doi:10.1016/j. biopsych.2015.03.025

29. Rong P, Liu J, Wang L, et al. Effect of transcutaneous auricular vagus nerve stimulation on major depressive disorder: a nonrandomized controlled pilot study. $J$ Affect Disord. 2016;195:172-179. doi:10.1016/j.jad.2016.02.031

30. Trevizol AP, Shiozawa P, Taiar I, et al. Transcutaneous vagus nerve stimulation (taVNS) for major depressive disorder: an open label proof-of-concept trial. Brain Stimul. 2016;9(3):453-454. doi:10.1016/j.brs.2016.02.001

31. Woodbury DM, Woodbury JW. Effects of vagal stimulation on experimentally induced seizures in rats. Epilepsia. 1990;31(Suppl 2):S7-S19. doi:10.1111/j.1528-1157.1990.tb05852.x

32. Zabara J. Inhibition of experimental seizures in canines by repetitive vagal stimulation. Epilepsia. 1992;33(6):1005-1012. doi:10. 1111/j.1528-1157.1992.tb01751.x

33. The Vagus Nerve Stimulation Study Group. A randomized controlled trial of chronic vagus nerve stimulation for treatment of medically intractable seizures. Neurology. 1995;45(2):224-230. doi:10.1212/wnl.45.2.224.

34. Handforth A, DeGiorgio CM, Schachter SC, et al. Vagus nerve stimulation therapy for partial-onset seizures: a randomized active-control trial. Neurology. 1998;51(1):48-55. doi:10.1212/wnl.51.1.48

35. Morris GL 3rd, Mueller WM. Long-term treatment with vagus nerve stimulation in patients with refractory epilepsy. The Vagus Nerve Stimulation Study Group E01-E05. Neurology. 1999;53 (8):1731-1735. doi:10.1212/wnl.53.8.1731

36. Sackeim HA, Decina P, Prohovnik I, Malitz S, Resor SR. Anticonvulsant and antidepressant properties of electroconvulsive therapy: a proposed mechanism of action. Biol Psychiatry. 1983;18(11):1301-1310.

37. Sackeim HA. The anticonvulsant hypothesis of the mechanisms of action of ECT: current status. J ECT. 1999;15(1):5-26. doi:10.1097/00124509-199903000-00003

38. Seymour J. Commentary and update on the contribution of the GABA hypothesis to understanding the mechanism of action of electroconvulsive therapy. $J$ ECT. 2020. doi:10.1097/YCT.000 0000000000711

39. Dunn R, Frye M, Kimbrell T, Denicoff K, Leverich G, Post R. The efficacy and use of anticonvulsants in mood disorders. Clin Neuropharmacol. 1998;21(4):215-235.

40. Reinares M, Rosa AR, Franco C, et al. A systematic review on the role of anticonvulsants in the treatment of acute bipolar depression. Int J Neuropsychopharmacol. 2012;16(2):485-496. doi:10.1017/s1461145712000491

41. Adair D, Truong D, Esmaeilpour Z, et al. Electrical stimulation of cranial nerves in cognition and disease. Brain Stimul. 2020;13 (3):717-750. doi:10.1016/j.brs.2020.02.019

42. Thompson N, Mastitskaya S, Holder D. Avoiding off-target effects in electrical stimulation of the cervical vagus nerve: neuroanatomical tracing techniques to study fascicular anatomy of the vagus nerve. J Neurosci Methods. 2019;325:108325. doi:10. 1016/j.jneumeth.2019.108325

43. Cottle MKW, Calaresu FR. Projections from the nucleus and tractus solitarius in the cat. J Comp Neurol. 1975;161 (2):143-157. doi:10.1002/cne.901610202

44. Ricardo JA, Tongju Koh E. Anatomical evidence of direct projections from the nucleus of the solitary tract to the hypothalamus, amygdala, and other forebrain structures in the rat. Brain Res. 1978;153(1):1-26. doi:10.1016/0006-8993(78)91125-3

45. Krahl SE, Senanayake SS, Pekary AE, Sattin A. Vagus nerve stimulation (VNS) is effective in a rat model of antidepressant action. J Psychiatr Res. 2004;38(3):237-240. doi:10.1016/j. jpsychires.2003.11.005 
46. Elger G, Hoppe C, Falkai P, Rush AJ, Elger CE. Vagus nerve stimulation is associated with mood improvements in epilepsy patients. Epilepsy Res. 2000;42(2-3):203-210. doi:10.1016/ s0920-1211(00)00181-9

47. Sackeim HA. The definition and meaning of treatment-resistant depression. J Clin Psychiatry. 2001;62(Suppl 16):10-17.

48. Sackeim HA, Aaronson ST, Bunker MT, et al. The assessment of resistance to antidepressant treatment: rationale for the antidepressant treatment history form: short form (ATHF-SF). J Psychiatr Res. 2019;113:125-136. doi:10.1016/j.jpsychires.20 19.03.021

49. Schlaepfer TE, Frick C, Zobel A, et al. Vagus nerve stimulation for depression: efficacy and safety in a European study. Psychol Med. 2008;38(5):651-661. doi:10.1017/S0033291707001924

50. Bajbouj M, Merkl A, Schlaepfer TE, et al. Two-year outcome of vagus nerve stimulation in treatment-resistant depression. $J$ Clin Psychopharmacol. 2010;30(3):273-281. doi:10.1097/JCP.0b013e $3181 \mathrm{db} 8831$

51. George MS, Rush AJ, Marangell LB, et al. A one-year comparison of vagus nerve stimulation with treatment as usual for treatment-resistant depression. Biol Psychiatry. 2005;58 (5):364-373. doi:10.1016/j.biopsych.2005.07.028

52. Rush AJ, Sackeim HA, Marangell LB, et al. Effects of 12 months of vagus nerve stimulation in treatment-resistant depression: a naturalistic study. Biol Psychiatry. 2005;58(5):355-363. doi:10. 1016/j.biopsych.2005.05.024

53. Depression Guideline Panel. Depression in primary care: Vol.2. Treatment of major depression. U.S. Department of Health and Human Services, Public Health Service, Agency for Health Care Policy and Research, AHCPR. No. 93-0551; 1993.

54. Labiner DM, Ahern GL. Vagus nerve stimulation therapy in depression and epilepsy: therapeutic parameter settings. Acta Neurol Scand. 2007;115(1):23-33. doi:10.1111/j.1600-0404.2006. 00732.x

55. Rush AJ, Gullion CM, Basco MR, Jarrett RB, Trivedi MH. The Inventory of Depressive Symptomatology (IDS): psychometric properties. Psychol Med. 1996;26(3):477-486. doi:10.1017/ S0033291700035558

56. Montgomery SA, Åsberg M. A new depression scale designed to be sensitive to change. $B r J$ Psychiatry. 1979;134:382-389. doi:10.1192/bjp.134.4.382

57. Guy W ECDEU assessment manual for psychopharmacology. Superintendent of Documents, U.S. Government Printing Office, U.S. Department of Health, Education, and Welfare Publication, No. 76-338; 1976.

58. Dunner DL, Rush AJ, Russell JM, et al. Prospective, long-term, multicenter study of the naturalistic outcomes of patients with treatment-resistant depression. $J$ Clin Psychiatry. 2006;67 (5):688-695. doi:10.4088/JCP.v67n0501

59. Post RM, Uhde TW, Rubinow DR, Huggins T. Differential time course of antidepressant effects after sleep deprivation, ECT, and carbamazepine: clinical and theoretical implications. Psychiatry Res. 1987;22(1):11-19. doi:10.1016/0165-1781(87)90045-X

60. Roy-Byrne PP, Uhde TW, Post RM. Effects of one night's sleep deprivation on mood and behavior in panic disorder. Patients with panic disorder compared with depressed patients and normal controls. Arch Gen Psychiatry. 1986;43(9):895-899. doi:10.10 01/archpsyc.1986.01800090085011

61. Rush AJ, Trivedi MH, Wisniewski SR, et al. Acute and longerterm outcomes in depressed outpatients requiring one or several treatment steps: a STAR*D report. Am J Psychiatry. 2006;163 (11):1905-1917. doi:10.1176/ajp.2006.163.11.1905

62. Conway CR, George MS, Sackeim HA. Towards an evidence-based, operational definition of treatment-resistant depression: when enough is enough. JAMA Psychiatry. 2017;74 (1):9-10. doi:10.1001/jamapsychiatry.2016.2586
63. Prudic J, Haskett RF, Mulsant B, et al. Resistance to antidepressant medications and short-term clinical response to ECT. Am J Psychiatry. 1996;153(8):985-992.

64. Prudic J, Sackeim HA, Devanand DP. Medication resistance and clinical response to electroconvulsive therapy. Psychiatry Res. 1990;31(3):287-296. doi:10.1016/0165-1781(90)90098-P

65. Heijnen WT, Birkenhager TK, Wierdsma AI, van den Broek WW. Antidepressant pharmacotherapy failure and response to subsequent electroconvulsive therapy: a meta-analysis. J Clin Psychopharmacol. 2010;30(5):616-619. doi:10.1097/JCP.0b013e3181ee0f5f

66. Sackeim HA, Prudic J, Devanand DP, Decina P, Kerr B, Malitz S. The impact of medication resistance and continuation pharmacotherapy on relapse following response to electroconvulsive therapy in major depression. J Clin Psychopharmacol. 1990;10 (2):96-104. doi:10.1097/00004714-199004000-00004

67. Sackeim HA, Prudic J, Devanand DP, et al. A prospective, randomized, double-blind comparison of bilateral and right unilateral electroconvulsive therapy at different stimulus intensities. Arch Gen Psychiatry. 2000;57(5):425-434. doi:10.1001/archpsyc.57. 5.425

68. Sackeim HA, Haskett RF, Mulsant BH, et al. Continuation pharmacotherapy in the prevention of relapse following electroconvulsive therapy: a randomized controlled trial. JAMA. 2001;285 (10):1299-1307. doi:10.1001/jama.285.10.1299

69. Prudic J, Haskett RF, McCall WV, et al. Pharmacological strategies in the prevention of relapse after electroconvulsive therapy. J ECT. 2013;29(1):3-12. doi:10.1097/YCT.0b013e31826ea8c4

70. Sackeim HA. Acute, continuation and maintenance treatment of major depressive episodes with Transcranial Magnetic Stimulation. Brain Stimul. 2016;9(3):313-319. doi:10.1016/j. brs.2016.03.006

71. Schultz D VNS therapy system PMA approval for chronic or recurrent depression. FDA. Available from: https://www.accessdata.fda. gov/cdrh_docs/pdf/P970003S050a.pdf. Accessed November 30, 2020.

72. Centers for Medicare and Medicaid Services. National coverage determination (NCD) for vagus nerve stimulation (VNS) (160.18). CMS; 2007. Available from: https://www.cms.gov/ Regulations-and-Guidance/Guidance/Transmittals/Downloads/ R70NCD.pdf. Accessed November 30, 2020.

73. NICE (National Institue for Health and Clinical Excellence). Implanted vagus nerve stimulation for treatment-resistant depression (IPG679). National Clinical Practice Guideline. NICE; 2020.

74. ANZHSN (Australai and New Zealand Horizon Scanning Network). National Horizon Scanning Unit Horizon Scanning Prioritising Summary: Update Number 3, VNS Therapy System for the Treatment of Depression. Adelaide Health Technology Assessment; 2007.

75. Aaronson ST, Carpenter LL, Conway CR, et al. Vagus nerve stimulation therapy randomized to different amounts of electrical charge for treatment-resistant depression: acute and chronic effects. Brain Stimul. 2013;6(4):631-640. doi:10.1016/j.brs.2012. 09.013

76. Sackeim HA, Prudic J, Devanand DP, et al. Effects of stimulus intensity and electrode placement on the efficacy and cognitive effects of electroconvulsive therapy. $N$ Engl J Med. 1993;328 (12):839-846. doi:10.1056/NEJM199303253281204

77. McCall WV, Reboussin DM, Weiner RD, Sackeim HA. Titrated moderately suprathreshold vs fixed high-dose right unilateral electroconvulsive therapy: acute antidepressant and cognitive effects. Arch Gen Psychiatry. 2000;57(5):438-444. doi:10.1001/ archpsyc.57.5.438

78. Sackeim HA, Prudic J, Nobler MS, et al. Effects of pulse width and electrode placement on the efficacy and cognitive effects of electroconvulsive therapy. Brain Stimul. 2008;1(2):71-83. doi:10.1016/j.brs.2008.03.001 
79. Panebianco M, Rigby A, Weston J, Marson AG. Vagus nerve stimulation for partial seizures. Cochrane Database Syst Rev. 2015;4:CD002896. doi:10.1002/14651858.CD002896.pub2

80. O'Reardon JP, Solvason HB, Janicak PG, et al. Efficacy and safety of transcranial magnetic stimulation in the acute treatment of major depression: a multisite randomized controlled trial. Biol Psychiatry. 2007;62(11):1208-1216. doi:10.1016/j.biopsych.2007.01.018

81. George MS, Lisanby SH, Avery D, et al. Daily left prefrontal transcranial magnetic stimulation therapy for major depressive disorder: a sham-controlled randomized trial. Arch Gen Psychiatry. 2010;67 (5):507-516. doi:10.1001/archgenpsychiatry.2010.46

82. Sackeim HA, Decina P, Prohovnik I, Malitz S. Seizure threshold in electroconvulsive therapy. Effects of sex, age, electrode placement, and number of treatments. Arch Gen Psychiatry. 1987;44 (4):355-360. doi:10.1001/archpsyc.1987.01800160067009

83. American Psychiatric Association. The Practice of ECT: Recommendations for Treatment, Training and Privileging. Second Edition. American Psychiatric Press; 2001.

84. Martin JLR, Martín-Sánchez E. Systematic review and meta-analysis of vagus nerve stimulation in the treatment of depression: variable results based on study designs. Eur Psychiatry. 2012;27(3):147-155. doi:10.1016/j.eurpsy.2011.07.006

85. Daban C, Martinez-Aran A, Cruz N, Vieta E. Safety and efficacy of Vagus Nerve Stimulation in treatment-resistant depression. A systematic review. $J$ Affect Disord. 2008;110(1):1-15. doi:10.1016/j.jad.2008.02.012

86. Lv H, Zhao Y-H, Chen J-G, Wang D-Y, Chen H. Vagus Nerve Stimulation for depression: a systematic review. Front Psychol. 2019;10:64. doi:10.3389/fpsyg.2019.00064

87. Gaynes BN, Asher G, Gartlehner G, et al. Definition of treatment-resistant depression in the medicare population. AHRQ technology assessments; 2018. Available from: https:// www.ncbi.nlm.nih.gov/pubmed/30260611. Accessed November 30,2020

88. Centers for Medicare and Medicaid Services. Decision memo for vagus nerve stimulation for treatment of resistant depression (TRD) (CAG-00313R). CMS; 2019. Available from: https:// www.cms.gov/medicare-coverage-database/details/nca-decisionmemo.aspx? ?CAId=292. Accessed November 30, 2020.

89. Conway CR, Olin B, Aaronson ST, et al. A prospective, multi-center randomized, controlled, blinded trial of vagus nerve stimulation for difficult to treat depression: a novel design for a novel treatment. Contemp Clin Trials. 2020;95:106066. doi:10.1016/j.cct.2020.106066

90. Murrough JW, Iosifescu DV, Chang LC, et al. Antidepressant efficacy of ketamine in treatment-resistant major depression: a two-site randomized controlled trial. Am J Psychiatry. 2013;170 (10):1134-1142. doi:10.1176/appi.ajp.2013.13030392
91. Canuso CM, Singh JB, Fedgchin M, et al. Efficacy and safety of Intranasal esketamine for the rapid reduction of symptoms of depression and suicidality in patients at Imminent risk for suicide: results of a double-blind, randomized, placebo-controlled study. Am J Psychiatry. 2018;175(7):620-630. doi:10.1176/appi.ajp.20 18.17060720

92. Nobler MS, Sackeim HA, Moeller JR, Prudic J, Petkova E, Waternaux C. Quantifying the speed of symptomatic improvement with electroconvulsive therapy: comparison of alternative statistical methods. Convuls Ther. 1997;13(4):208-221.

93. Lapidus KA, Shin JS, Pasculli RM, Briggs MC, Popeo DM, Kellner CH. Low-dose right unilateral electroconvulsive therapy (ECT): effectiveness of the first treatment. $J$ ECT. 2013;29 (2):83-85. doi:10.1097/YCT.0b013e31827e0b51

94. Machado-Vieira R, Baumann J, Wheeler-Castillo C, et al. The timing of antidepressant effects: a comparison of diverse pharmacological and somatic treatments. Pharmaceuticals (Basel, Switzerland). 2010;3(1):19-41. doi:10.3390/ph3010019

95. Rodrigues FB, Duarte GS, Prescott D, Ferreira J, Costa J. Deep brain stimulation for dystonia. Cochrane Database Syst Rev. 2019;1:CD012405. doi:10.1002/14651858.CD012405.pub2

96. Vitek JL, Jain R, Chen L, et al. Subthalamic nucleus deep brain stimulation with a multiple independent constant currentcontrolled device in Parkinson's disease (INTREPID): a multicentre, double-blind, randomised, sham-controlled study. Lancet Neurol. 2020;19(6):491-501. doi:10.1016/S1474-4422 (20)30108-3

97. Salanova V, Witt T, Worth R, et al. Long-term efficacy and safety of thalamic stimulation for drug-resistant partial epilepsy. Neurology. 2015;84(10):1017-1025. doi:10.1212/WNL.00000000 00001334

98. Fisher R, Salanova V, Witt T, et al. Electrical stimulation of the anterior nucleus of thalamus for treatment of refractory epilepsy. Epilepsia. 2010;51(5):899-908. doi:10.1111/j.1528-1167.2010.02 536.x

99. Schruers K, Baldi S, van den Heuvel T, et al. The effects of deep-brain non-stimulation in severe obsessive-compulsive disorder: an individual patient data meta-analysis. Transl Psychiatry. 2019;9(1):183. doi:10.1038/s41398-019-0522-6

100. Paykel ES. Remission and residual symptomatology in major depression. Psychopathology. 1998;31(1):5-14. doi:10.1159/ 000029018

101. Paykel ES. Achieving gains beyond response. Acta Psychiatr Scand Suppl. 2002;415:12-17. doi:10.1034/j.1600-0447.106.s4 15.3.x

102. Bloch MH, Green C, Kichuk SA, et al. Long-term outcome in adults with obsessive-compulsive disorder. Depress Anxiety. 2013;30(8):716-722. doi:10.1002/da.22103
Neuropsychiatric Disease and Treatment

\section{Publish your work in this journal}

Neuropsychiatric Disease and Treatment is an international, peerreviewed journal of clinical therapeutics and pharmacology focusing on concise rapid reporting of clinical or pre-clinical studies on a range of neuropsychiatric and neurological disorders. This journal is indexed on PubMed Central, the 'PsycINFO' database and CAS, an is the official journal of The International Neuropsychiatric Association (INA). The manuscript management system is completely online and includes a very quick and fair peer-review system, which is all easy to use. Visit http://www.dovepress.com/testimonials.php to read real quotes from published authors. 